\title{
Van der Waals coefficients for systems with ultracold polar alkali-metal molecules
}

\author{
P. S. Żuchowski, ${ }^{1, *}$ M. Kosicki, ${ }^{2}$ M. Kodrycka, ${ }^{2}$ and P. Soldán ${ }^{3, \dagger}$ \\ ${ }^{1}$ Institute of Physics, Faculty of Physics, Astronomy and Informatics, \\ Nicolaus Copernicus University, Grudziadzka 5, 87-100 Torun, Poland \\ ${ }^{2}$ Physics Institute, Kazimierz Wielki University, pl. Weysenhoffa 11, 85-072 Bydgoszcz, Poland \\ ${ }^{3}$ Department of Chemical Physics and Optics, Faculty of Mathematics and Physics, \\ Charles University in Prague, Ke Karlovu 3, CZ-12116 Prague 2, Czech Republic
}

\begin{abstract}
A systematic study of the leading isotropic van der Waals coefficients for the alkali-metal atom + molecule and molecule + molecule systems is presented. Dipole moments and static and dynamic dipole polarizabilities are calculated employing high-level quantum chemistry calculations. The dispersion, induction, and rotational parts of the isotropic van der Waals coefficient are evaluated. The known van der Waals coefficients are then used to derive characteristics essential for simple models of the collisions involving the corresponding ultracold polar molecules.
\end{abstract}

PACS numbers: $34.50 . \mathrm{Cx}, 31.50 . \mathrm{Bc}$

\section{INTRODUCTION}

Ultracold chemistry in sub-microkelvin regime has emerged as one of the most exciting fields in atomic and molecular physics [1-3]. By tuning magnetic field across Feshbach resonances one can combine two free atoms into a bound state, with binding energy of order of $\mathrm{MHz}$ and then, with elaborated laser techniques, coherently transfer them into the deeply bound states - including the absolute rotational-vibrational-electronic ground state. At present two alkali-metal dimers have been produced in this manner $\mathrm{KRb}$ and $\mathrm{Cs}_{2}[4,5]$. It is also worth mentioning that LiCs molecules in the vibrational ground state have been produced by photoassociation followed by spontaneous emission [6]. At present, many experimental groups have focused on production of other heteronuclear alkali-metal dimers hoping to obtain ultracold quantum gases of polar molecules, stable with respect to the atom exchange and trimer formation [7]. Such quantum gases of polar molecules will be used to explore new ideas in quantum information theory $[8,9]$, quantum simulations of condensed-phase physics [10], or fundamental studies of chemical reactions [11].

Description of chemical processes in sub-microkelvin regime is extremely difficult, because the full quantum calculation for such systems is nearly impossible. Thus only few quantum dynamics studies of ultracold atom+diatom collisions employing global potential energy surfaces have been performed so far concentrating on the homonuclear spin-polarized systems [12-18], where single-electronic-state approach provides good approximation [19]. Even then, the quantum dynamics calculations for heavier system are very challenging and have not been yet performed despite the fact that the corresponding quartet potential energy surfaces are rather simple $[20,21]$.

\footnotetext{
* pzuch@fizyka.umk.pl

$\dagger$ corresponding author: pavel.soldan@mff.cuni.cz
}

Theoretical treatment of non-spin-polarized systems would be even more challenging. The calculations of triatomic and tetraatomic interaction potentials in such a case would have to include many active electrons and coupled potential energy surfaces, which at present is very far from routine. The following quantum dynamics calculations, especially in the presence of external fields, would be extremely demanding. The interaction potentials involving alkali-metal atoms and dimers are likely to be strongly anisotropic, and therefore the basis sets for such calculations would have to be very large. On the other hand, there is a very small number of observables as outcome of ultracold collisions. After all, in a laboratory we do not record state-resolved cross sections but only loss rates from the state prepared before the experiment. Thus, the recent theories of ultracold collisions [22-25], formulated to explain current experiments in this field, use only few simple parameters that catch the essential physics. Importantly enough, the feature of the intermolecular interaction that matters the most is the long-range shape of the interaction potential, usually represented analytically by the well-known van der Waals expansion with the most important term $-C_{6} R^{-6}(R$ is the distance between the monomer centres of mass).

Properties of the alkali-metal dimers have been intensively studied using electronic structure methods. A systematic study of the dipole moments of all possible alkalimetal dimers was published by Aymar and Dulieu [26], and Deiglmeyr et al. [27] reported a systematic study of the static dipole polarizabilities for these systems. Their approach, based on large effective core potentials combined with appropriately set core-polarization potentials, was particularly successful in predicting binding energies and spectroscopic properties of the alkali-metal dimers in the ground and low-lying excited states.

In this paper we report a systematic $a b$ initio study of the isotropic van der Waals $C_{6}$ coefficients for the alkali-metal atom + molecule $(\mathrm{A}+\mathrm{AB})$ and molecule + molecule $(\mathrm{AB}+\mathrm{AB})$ systems. We also derive characteristics essential for simple models of the corresponding ultra-low-energy collisions. In the following calculations 
masses of the bosonic ${ }^{7} \mathrm{Li},{ }^{23} \mathrm{Na},{ }^{41} \mathrm{~K},{ }^{87} \mathrm{Rb}$, and ${ }^{133} \mathrm{Cs}$ isotopes were used.

\section{METHODOLOGY}

The purpose of this paper is to provide essential parameters for modelling of collisions between the polar molecules in their ground rovibrational state. If colliding molecules are in $j=0$ states only isotropic part of the interaction potential governs its scattering properties at very long range - larger than $R_{\mathrm{vdW}}=\left(2 m C_{6} / \hbar^{2}\right)^{\frac{1}{4}}$, where $m$ is the reduced mass of the colliding system. If the strength of the anisotropy of the potential becomes comparable with spacing of the appropriate rotational energy levels of the molecule, then anisotropic term becomes important: for example in case of $\mathrm{A}+\mathrm{AB}$ collisions, the coupling driven by $C_{62}$ between $j=0$ and $j=2$ channels becomes important if the potential anisotropy is comparable with $6 \mathrm{~B}$. The same argument holds also for the $\mathrm{AB}+\mathrm{AB}$ collisions.

It is well known $[28,29]$ that within the BornOppenheimer approximation the isotropic van der Waals $C_{6}$ coefficient of a two-monomer system $(\mathrm{X}+\mathrm{Y})$ can be decomposed into two contributions each corresponding to a different effect. First, it contains a dispersion contribution $C_{6}^{\text {disp }}$ that physically represents the interaction of fluctuating instantaneous dipole moments, which are due to the movements of electrons, which correlate between interacting species at long range. Secondly, in the case of the heteronuclear dimers, a permanent molecular dipole moment induces a dipole moment on the atom, which in turn interacts with the permanent molecular dipole moment. This induction contribution $C_{6}^{\text {ind }}$ is usually smaller than the dispersion contribution.

The dispersion contribution to the isotropic van der Waals $C_{6}$ coefficient can be calculated from the following integral

$$
C_{6}^{\text {disp }}=\frac{3}{\pi} \int_{0}^{\infty} \bar{\alpha}_{\mathrm{X}}(i \omega) \bar{\alpha}_{\mathrm{Y}}(i \omega) d \omega
$$

where $i$ is the unit imaginary number, $\omega$ is frequency, and

$$
\bar{\alpha}_{\mathrm{mol}}(i \omega)=\frac{1}{3}\left[\alpha_{\mathrm{xx}}(i \omega)+\alpha_{\mathrm{yy}}(i \omega)+\alpha_{\mathrm{zz}}(i \omega)\right]
$$

is the orientation-averaged molecular dynamic dipole polarizability. The induction contribution to the isotropic van der Waals $C_{6}$ coefficient can be expressed as

$$
C_{6}^{\text {ind }}=\mu_{X}^{2} \bar{\alpha}_{\mathrm{Y}}(0)+\mu_{Y}^{2} \bar{\alpha}_{\mathrm{X}}(0),
$$

where $\mu$ is the corresponding permanent molecular dipole moment. If the monomer $\mathrm{X}$ is an atom in the sphericalsymmetry ground state and the overlap of the charge distribution of interacting species can be neglected, the first term in the above equation vanishes. The total isotropic $C_{6}$ coefficient of the atom + molecule system is a sum of the dispersion and induction contributions.
For molecule + molecule systems in their ground rotational state there also exists a (non-Born-Oppenheimer) rotational contribution to the effective isotropic $C_{6}$ resulting from a second-order coupling of the dipole-dipole term [30-32]. It which has the form $C_{6}^{\text {rot }}=\mu^{4} / 6 B$ where $B$ is the molecule rotational constant. Then the total isotropic $C_{6}$ coefficient for the molecule + molecule system is a sum of the dispersion, induction, and rotational contributions.

Proper choice of the electron basis set is crucial for quantum chemistry calculations of the dipole moments and polarizabilities. For lithium and sodium atoms we have used available core-valence correlation-consistent basis sets cc-pCV5Z designed by Prascher et al. [33], which we augmented by one set of diffuse functions. Effective-core potentials (ECPs) with tailored valence basis sets for heavy (K - Fr) alkali metal atoms have been optimized by Lim et al. [34]. These ECPs are smallcore type potentials, i.e. the outermost 9 electrons are described explicitly. To eliminate possible errors due to the basis incompleteness we have improved the original valence basis sets by adding $g$ and $h$ functions. and augmenting the basis sets by one set of diffuse functions. These basis sets have been tested on the atomic static dipolar polarizabilities, which have been calculated with the spin-restricted open-shell coupled cluster method [35] with single, double and non-iterative triple excitations $[\operatorname{RCCSD}(\mathrm{T})]$ employing a finite-field approach. In all cases the agreement with the reference values of Derevianko et al. [36] was very good (the difference for $\mathrm{Na}$ was 2.2 a.u.; less than one atomic unit for other alkali metals).

All alkali-metal dimers in their ground electronic state $X^{1} \Sigma^{+}$(near their equilibrium lengths) have their excited states significantly separated in energy, thus we can properly describe them by a single-reference Slater determinant, which is ideal for using the coupled cluster approach [35]. For the molecular calculations we took the equilibrium distances, which were optimized by the Paris group [26, 27].

For the molecular dynamic polarizability calculations we employed the time-independent coupled cluster polarization propagator method in singles- and doubles approximation (TI-CCSD). This was introduced by Moszynski et al. [37] and implemented in Molpro 2010.2 program [38]. Several approximations to the full timeindependent polarization propagator were discussed by Korona et al. [39]. In our study, we used the so-called $\operatorname{CCSD}(3)$ approximation of the TI-CCSD method, which is exact to the third order of the electronic correlation operator. In benchmark calculations against the dynamic dipole polarizabilities based on the full-configurationinteraction response functions, the $\operatorname{CCSD}(3)$ approximation demonstrated systematically a smaller error than the other approximations introduced there [37, 39]. Finally, in this paper we have used a finite-field $\operatorname{CCSD}(\mathrm{T})$ approach in order to evaluate dipole moments and static dipole polarizabilities of the alkali-metal dimers. Such 
calculations were also needed to verify the accuracy of the TI-CCSD dynamic polarizabilities.

\section{RESULTS AND DISCUSSION}

The dipole moments of the heteronuclear alkali-metal dimers calculated with the finite-filed $\operatorname{CCSD}(\mathrm{T})$ method as the first derivatives of energy with respect to the electric field applied are collected in Table I). The FF$\operatorname{CCSD}(\mathrm{T})$ data are in a good agreement with those obtained by Aymar and Dulieu [26], with an error of at most $10 \%$ for LiNa and KRb molecules - note that for these species the dipole moment and charge separation between atoms is significantly smaller than in other cases and the corresponding dipole moments are small. The dipole moment we have obtained for $\mathrm{KRb}(0.62 \mathrm{D})$ is somewhat larger than the experimental value $(0.566 \pm 0.017 \mathrm{D}$ [4]) and the value in Ref. [32]. For the sake of consistency we used our calculated values in further calculations. In addition to the finite-field $\operatorname{CCSD}(\mathrm{T})$ values we also calculated the finite-field CCSD values in order to check how important is the inclusion of triply excited configurations in calculations of the alkali-metal dimer dipole moments. The FF-CCSD values are also in good agreement with our reference FF-CCSD(T) data (the FF-CCSD values are systematically higher by $10 \%$ ). The fact that the triples contribution to the dipole moments is not too substantial indicates that the FF-CCSD(T) result might be very close to real values as the expansion of the molecular wavefunction in terms of number of excitations should converge rather quickly.

In order to verify the quality of the molecular dynamic dipole polarizabilities calculated with TI-CCSD we performed further tests by checking their values in the static limit against the polarizabilities calculated with the FF$\operatorname{CCSD}(\mathrm{T})$ approach and literature data. As the reference values we have used those published by Deiglmayr et al. [27] who used a 2-electron full configuration interaction method with carefully tailored large-core effective core potentials including core polarization potentials. This approach has proven to be accurate, for example, in predicting experimental values of the dipole moments of KRb [4], LiCs [40], and transition dipole moments RbCs [41]. We have also used for comparison the values of Urban and Sadlej [42], which were obtained with entirely different approach - using all-electron basis sets with scalar relativistic effects included. Our finite-field results agree very well with the results from Refs. [27, 42]; our FF-CCSD $(T)$ values of the orientation-averaged molecular static dipole polarizability are systematically right in between their values with differences not exceeding $6 \%$. The agreement between our TI-CCSD and FF-CCSD $(\mathrm{T})$ values of the orientation-averaged molecular static dipole polarizability is even better (see Table I). With the exception of LiNa, where the difference is indeed negligible, the TI-CCSD values are systematically higher than the FF-CCSD $(T)$ values with the differences never exceeding
$2.5 \%$. The anisotropy of the molecular static dipole polarizability $\Delta \alpha_{\mathrm{mol}}(0)$ exhibits the same tendency. With the exception of LiNa the TI-CCSD values are systematically higher than the FF-CCSD $(\mathrm{T})$ values with the differences ranging from $2.5 \%$ to $13 \%$.

In the evaluation of formulas (1) and (3) we used the TI-CCSD values of the molecular dynamic dipole polarizabilities, the FF-CCSD(T) values of the molecular dipole moments and molecular static dipole polarizabilities, and the values of the atomic static and dynamic polarizabilities from Ref. [36]. The integral in Eq. (1) was evaluated using Gauss quadrature for 50 frequencies also provided by Derevianko et al. [36].

Tables II and III contain the predicted isotropic van der Waals $C_{6}$ coefficients for the $\mathrm{A}+\mathrm{AB}$ and $\mathrm{AB}+\mathrm{AB}$ systems, respectively. In the case of the $A+A B$ systems there is a very clear progression in increase of the $C_{6}$ coefficient for both A and B from Li toward Cs. The induction contribution to $C_{6}$ is usually small; only in the case of significantly polar LiCs, LiRb, NaCs, and $\mathrm{NaRb}$ molecules it is within the range $10-23 \%$. Our $C_{6}$ values for the $\mathrm{K}+\mathrm{KRb}, \mathrm{Rb}+\mathrm{KRb}, \mathrm{Rb}+\mathrm{RbCs}$, and $\mathrm{Cs}+\mathrm{RbCs}$ systems are systematically larger than those reported by Kotochigova [24] by $8 \%, 6 \%, 35 \%$, and $41 \%$, respectively. The result for $\mathrm{KRb}+$ atom is clearly in good agreement with the result of Kotochigova, however, the difference for RbCs+atoms is significantly larger. The dynamic polarizability in Ref. [24] has been obtained as a sumover-state with appropriate transition dipole moments of the RbCs molecule. It is likely that this way the $\mathrm{RbCs}$ dynamic polarizability might have been underestimated using such procedure by neglecting some contributions or underestimating the continuum contribution. Note also, that the induction contribution for atom+diatom has not been included in Ref. [24].

It was proposed recently to approximate the $C_{6}$ coefficients by simply adding the pairwise atom-atom $C_{6}$ coefficients $[24,43]$. Our calculations have verified this model as seemingly reasonably good for heavy atoms (Cs and $\mathrm{Rb}$ ) interacting with weakly polar molecules. This nice agreement is, however, fortuitous, since this additive approximation includes only dispersion and no induction . In this case, the approximation of the trimer dispersion forces by simply adding them among dimers overcompensates the lack of the induction interaction.

As expected, the effective isotropic $C_{6}$ coefficients for the $A B+A B$ systems very strongly depend on the $A B$ dipole moment. Only the LiNa and KRb dimers with smallest dipole moments are dominated by electronic contribution to the $C_{6}$ coefficient, in other cases rotational contribution completely dominates the total $C_{6}$ coefficient. For the $\mathrm{AB}+\mathrm{AB}$ systems, there is also a very distinct pattern in increase of the electronic contribution similar to the $\mathrm{A}+\mathrm{AB}$ systems. The $\mathrm{KRb}$ isotropic $C_{6}$ coefficient is higher by $10 \%$ compared to the value of Kotochigova [24] and by $6 \%$ with respect to the value given by Buchachenko and coworkers [32]. Our results are in agreement with those reported by Quéméner et al. [25] 
TABLE I. Dipole moments (in Debye), rotational constant $\left(\right.$ in $\mathrm{cm}^{-1}$ ), the orientation-averaged molecular static dipole polarizability (in atomic units), and the anisotropy of the molecular static dipole polarizability (in atomic units) of the ground $X^{1} \Sigma^{+}$ states of heteronuclear alkali-metal dimers calculated at the equilibrium interatomic distances from Ref. [26].

\begin{tabular}{|c|c|c|c|c|c|c|c|}
\hline dimer & $r_{e} / a_{0}[26]$ & $B / h c$ & $\mu$ & $\overline{\bar{\alpha}_{\mathrm{mol}}^{\mathrm{FF}}(0)}$ & $\bar{\alpha}_{\mathrm{mol}}^{\mathrm{TI}}(0)$ & $\Delta \alpha_{\mathrm{mol}}^{\mathrm{FF}}(0)$ & $\Delta \alpha_{\mathrm{mol}}^{\mathrm{TI}}(0)$ \\
\hline $\mathrm{LiNa}$ & 5.4518 & 0.425 & 0.48 & 237.7 & 237.6 & 156.3 & 155.7 \\
\hline $\mathrm{LiK}$ & 6.268 & 0.293 & 3.41 & 324.2 & 326.9 & 234.5 & 240.7 \\
\hline $\mathrm{LiRb}$ & 6.5 & 0.254 & 3.99 & 347.2 & 352.1 & 262.0 & 272.7 \\
\hline LiCs & 6.93 & 0.218 & 5.39 & 391.9 & 399.1 & 317.8 & 333.1 \\
\hline $\mathrm{NaK}$ & 6.61 & 0.094 & 2.72 & 358.1 & 362.7 & 247.2 & 260.9 \\
\hline $\mathrm{NaRb}$ & 6.88 & 0.070 & 3.31 & 387.1 & 393.9 & 279.2 & 299.7 \\
\hline $\mathrm{NaCs}$ & 7.27 & 0.058 & 4.63 & 439.3 & 448.0 & 339.4 & 364.1 \\
\hline $\mathrm{KRb}$ & 7.688 & 0.037 & 0.62 & 523.5 & 532.3 & 367.6 & 409.5 \\
\hline $\mathrm{KCs}$ & 8.095 & 0.030 & 1.98 & 596.0 & 606.8 & 436.1 & 488.9 \\
\hline $\mathrm{RbCs}$ & 8.366 & 0.017 & 1.32 & 638.6 & 653.0 & 462.1 & 531.1 \\
\hline
\end{tabular}

TABLE II. The isotropic $C_{6}$ van der Waals coefficients (in atomic units) for the alkali-metal $\mathrm{A}+\mathrm{AB}$ systems. The last column shows the value based on pair-wise atom-atom additive model.

\begin{tabular}{llrrrr}
\hline \hline atom & dimer & $C_{6}^{\text {disp }}$ & $C_{6}^{\text {ind }}$ & $C_{6}$ & $C_{6}^{\text {add }}$ \\
\hline $\mathrm{Li}$ & $\mathrm{LiNa}$ & 2217 & 6 & 2223 & 2856 \\
& $\mathrm{LiK}$ & 2885 & 294 & 3179 & 3711 \\
& $\mathrm{LiRb}$ & 3098 & 407 & 3505 & 3934 \\
& $\mathrm{LiCs}$ & 3452 & 740 & 4192 & 4454 \\
\hline $\mathrm{Na}$ & $\mathrm{LiNa}$ & 2358 & 6 & 2364 & 3023 \\
& $\mathrm{NaK}$ & 3405 & 187 & 3592 & 4003 \\
& $\mathrm{NaRb}$ & 3673 & 275 & 3948 & 4239 \\
& $\mathrm{NaCs}$ & 4092 & 539 & 4631 & 4783 \\
\hline $\mathrm{K}$ & $\mathrm{LiK}$ & 4821 & 520 & 5341 & 6219 \\
& $\mathrm{NaK}$ & 5364 & 334 & 5698 & 6344 \\
& $\mathrm{KRb}$ & 7428 & 17 & 7445 & 8171 \\
& $\mathrm{KCs}$ & 8298 & 175 & 8473 & 9056 \\
\hline $\mathrm{Rb}$ & $\mathrm{LiRb}$ & 5688 & 790 & 6478 & 7235 \\
& $\mathrm{NaRb}$ & 6357 & 539 & 6896 & 7373 \\
& $\mathrm{KRb}$ & 8154 & 19 & 8173 & 8964 \\
& $\mathrm{RbCs}$ & 9751 & 87 & 9838 & 10353 \\
\hline $\mathrm{Cs}$ & $\mathrm{LiCs}$ & 7652 & 1803 & 9455 & 9911 \\
& $\mathrm{NaCs}$ & 8555 & 1324 & 9879 & 10073 \\
& $\mathrm{KCs}$ & 10995 & 242 & 11237 & 12005 \\
& $\mathrm{RbCs}$ & 11772 & 110 & 11882 & 12509 \\
\hline \hline
\end{tabular}

TABLE III. The isotropic $C_{6}$ van der Waals coefficients (in atomic units) for the alkali-metal $\mathrm{AB}+\mathrm{AB}$ systems.

\begin{tabular}{llrrr}
\hline \hline dimer & $C_{6}^{\text {disp }}$ & $C_{6}^{\text {ind }}$ & $C_{6}^{\text {rot }}$ & $C_{6}$ \\
\hline $\mathrm{LiNa}$ & 3582 & 17 & 110 & 3709 \\
$\mathrm{LiK}$ & 6024 & 1167 & 404491 & 411682 \\
$\mathrm{LiRb}$ & 6963 & 1711 & 876031 & 884705 \\
$\mathrm{LiCs}$ & 8670 & 3520 & 3397216 & 3409406 \\
\hline $\mathrm{NaK}$ & 7461 & 820 & 508325 & 516606 \\
$\mathrm{NaRb}$ & 8696 & 1313 & 1497080 & 1507089 \\
$\mathrm{NaCs}$ & 10822 & 2916 & 6932958 & 6946696 \\
\hline $\mathrm{KRb}$ & 14202 & 62 & 3456 & 17720 \\
$\mathrm{KCs}$ & 17716 & 723 & 450681 & 469120 \\
\hline $\mathrm{RbCs}$ & 20301 & 345 & 160336 & 180982 \\
\hline \hline
\end{tabular}

for the $\mathrm{LiNa}$ (difference of $4 \%$ ), $\mathrm{LiK}(20 \%)$, LiRb (17\%), and $\mathrm{LiCs}(11 \%)$ systems. These values are very sensitive to the dipole moment and rotational constant of the molecule, thus even small differences in these characteristics can easily translate into a $20 \%$ difference in the dominating rotational part of the $C_{6}$ coefficient.

Known $C_{6}$ coefficients allow us to determine the energy limits for single partial-wave scattering. The p-wave or $\mathrm{d}$-wave scattering starts to dominate if the collision energy is comparable to the appropriate centrifugal barrier heights: for the $\mathrm{A}+\mathrm{AB}$ collisions it is the p-wave, while for the bosonic $\mathrm{AB}+\mathrm{AB}$ collisions it is the d-wave scattering. In Table IV we have included the centrifugal barrier heights for the $\mathrm{A}+\mathrm{AB}$ scattering and bosonic $\mathrm{AB}+\mathrm{AB}$ scattering. Their values approximately determine the single partial-wave regime. The same table contains also the mean scattering lengths [44], which illustrate, in a sense, a characteristic length scale of the corresponding interaction potential.

\section{CONCLUSIONS}

In conclusion, we have reported a complete $a b$ initio study of the isotropic $C_{6}$ van der Waals coefficients in all possible $\mathrm{A}+\mathrm{AB}$ and $\mathrm{AB}+\mathrm{AB}$ systems, where $\mathrm{A}$ and $\mathrm{B}$ are two distinct alkali-metal atoms and $\mathrm{AB}$ are molecules are in their ground state. Given the rapid development of the field and many ongoing experiments with polar alkalimetal molecules, we expect that these results will be beneficial for modelling their collisional properties, which are crucial for stability studies of the ultracold molecular dipolar gases in traps. In future studies we would like to pay increased attention to the role of anisotropy in ultracold collisions, and we would also like to exploit our results when constructing potential energy surfaces for various collisional systems. 
TABLE IV. Heights (in $\mu K$ ) of p-wave centrifugal barriers $V_{\mathrm{p}}$ for the $\mathrm{A}+\mathrm{AB}$ systems and d-wave centrifugal barriers $V_{\mathrm{d}}$ for the $\mathrm{AB}+\mathrm{AB}$ systems, respectively, with mean scattering lengths $\bar{a}$ (in $a_{0}$ ) for the corresponding collisions.

\begin{tabular}{llcc|lcc}
\hline \hline atom & dimer & $V_{\mathrm{p}}$ & $\bar{a}$ & dimer & $V_{\mathrm{d}}$ & $\bar{a}$ \\
\hline $\mathrm{Li}$ & $\mathrm{LiNa}$ & 2442 & 39 & LiNa & 2293 & 30 \\
& $\mathrm{LiK}$ & 1844 & 44 & $\mathrm{LiK}$ & 111 & 110 \\
& $\mathrm{LiRb}$ & 1581 & 46 & $\mathrm{LiRb}$ & 28 & 157 \\
& $\mathrm{LiCs}$ & 1397 & 48 & $\mathrm{LiCs}$ & 8 & 244 \\
\hline $\mathrm{Na}$ & $\mathrm{LiNa}$ & 684 & 49 & & & \\
& $\mathrm{NaK}$ & 380 & 58 & $\mathrm{NaK}$ & 64 & 125 \\
& $\mathrm{NaRb}$ & 300 & 61 & $\mathrm{NaRb}$ & 16 & 187 \\
& $\mathrm{NaCs}$ & 256 & 65 & $\mathrm{NaCs}$ & 6 & 251 \\
\hline $\mathrm{K}$ & $\mathrm{LiK}$ & 221 & 68 & & & \\
& $\mathrm{NaK}$ & 177 & 71 & & & \\
& $\mathrm{KRb}$ & 112 & 81 & $\mathrm{KRb}$ & 119 & 64 \\
& $\mathrm{KCs}$ & 95 & 85 & $\mathrm{KCs}$ & 15 & 155 \\
\hline $\mathrm{Rb}$ & $\mathrm{LiRb}$ & 64 & 86 & & & \\
& $\mathrm{NaRb}$ & 56 & 89 & & & \\
& $\mathrm{KRb}$ & 47 & 95 & & & \\
& $\mathrm{RbCs}$ & 32 & 104 & $\mathrm{RbCs}$ & 17 & 131 \\
\hline $\mathrm{Cs}$ & $\mathrm{LiCs}$ & 29 & 105 & & & \\
& $\mathrm{NaCs}$ & 26 & 108 & & & \\
& $\mathrm{KCs}$ & 23 & 112 & & & \\
& $\mathrm{RbCs}$ & 19 & 117 & & & \\
\hline \hline
\end{tabular}

\section{ACKNOWLEDGEMENTS}

P.S.Z., M.K., and M.K. acknowledge funding from the Homing Plus programme (Project No. 2011-3/14) of the Foundation for Polish Science, which is co-financed by the European Regional Development Fund of the European Union. We are also grateful for the computer time from the Wroclaw Centre for Networking and Supercomputing.
[1] J. M. Hutson and P. Soldán, Int. Rev. Phys. Chem. 25, 497 (2006).

[2] L. Carr, D. DeMille, R. Krems, and J. Ye, New J. Phys. 11, 055049 (2009).

[3] J. Ulmanis, J. Deiglmayr, M. Repp, R. Wester, and M. Weidemuller, Chem. Rev. 112, 4890 (2012).

[4] K.-K. Ni, S. Ospelkaus, M. H. G. de Miranda, A. Pe'er, B. Neyenhuis, J. J. Zirbel, S. Kotochigova, P. S. Julienne, D. S. Jin, and J. Ye, Science 322, 231 (2008).

[5] J. G. Danzl, E. Haller, M. Gustavsson, M. J. Mark, R. Hart, N. Bouloufa, O. Dulieu, H. Ritsch, and H.-C. Nägerl, Science 321, 1062 (2008).

[6] J. Deiglmayr, A. Grochola, M. Repp, K. Mörtlbauer, C. Glück, J. Lange, O. Dulieu, R. Wester, and M. Weidemüller, Phys. Rev. Lett. 101, 133004 (2008).

[7] P. S. Żuchowski and J. M. Hutson, Phys. Rev. A 81, 060703 (2010).

[8] D. DeMille, Phys. Rev. Lett. 88, 067901 (2002).

[9] P. Rabl, D. DeMille, J. M. Doyle, M. D. Lukin, R. J. Schoelkopf, and P. Zoller, Phys. Rev. Lett. 97, 033003 (2006).

[10] H. P. Büchler, E. Demler, M. Lukin, A. Micheli, N. Prokof'ev, G. Pupillo, and P. Zoller, Phys. Rev. Lett. 98, 060404 (2007).

[11] S. Ospelkaus, K.-K. Ni, D. Wang, M. H. G. de Miranda, B. Neyenhuis, G. Quéméner, P. S. Julienne, J. L. Bohn, D. S. Jin, and J. Ye, Science 327, 853 (2010).

[12] P. Soldán, M. T. Cvitaš, J. M. Hutson, P. Honvault, and J. M. Launay, Phys. Rev. Lett. 89, 153201 (2002).

[13] G. Quéméner, P. Honvault, and J. M. Launay, Eur. Phys. J. D 30, 201 (2004).
[14] M. T. Cvitaš, P. Soldán, J. M. Hutson, P. Honvault, and J. M. Launay, Phys. Rev. Lett. 94, 033201 (2005).

[15] M. T. Cvitaš, P. Soldán, J. M. Hutson, P. Honvault, and J. M. Launay, Phys. Rev. Lett. 94, 200402 (2005).

[16] G. Quéméner, P. Honvault, J. M. Launay, P. Soldán, D. E. Potter, and J. M. Hutson, Phys. Rev. A 71, 032722 (2005).

[17] M. T. Cvitaš, P. Soldán, J. M. Hutson, P. Honvault, and J. M. Launay, J. Chem. Phys. 127, 074302 (2007).

[18] A. Simoni, J.-M. Launay, and P. Soldán, Phys. Rev. A 79, 032701 (2009).

[19] J. M. Hutson and P. Soldán, Int. Rev. Phys. Chem. 26, 1 (2007).

[20] R. Guérout, P. Soldán, M. Aymar, J. Deiglmayr, and O. Dulieu, Int. J. Quantum Chem. 109, 3387 (2009).

[21] P. Soldán, J. Chem. Phys. 132, 234308 (2010).

[22] Z. Idziaszek and P. S. Julienne, Phys. Rev. Lett. 104, 113202 (2010).

[23] Z. Idziaszek, G. Quéméner, J. L. Bohn, and P. S. Julienne, Phys. Rev. A 82, 020703R (2010).

[24] S. Kotochigova, New Journal of Physics 12, 073041 (2010).

[25] G. Quéméner, J. L. Bohn, , A. Petrov, and S. K. Kotochigova, Phys. Rev. A 84, 062703 (2011).

[26] M. Aymar and O. Dulieu, J. Chem. Phys. 122, 204302 (2005).

[27] J. Deiglmayr, M. Aymar, O. Dulieu, R. Wester, and M. Weidemüller, J. Chem. Phys. 129, 064309 (2008).

[28] A. Stone, The theory of intermolecular forces (Clarendon Press, Oxford, 2002).

[29] I. Kaplan, Intermolecular interactions (John Wiley \& Sons, Chichester, 2006). 
[30] A. V. Avdeenkov and J. L. Bohn, Phys. Rev. A 66, 052718 (2002).

[31] R. Barnett, D. Petrov, M. Lukin, and E. Demler, Phys. Rev. Lett. 96, 190401 (2006).

[32] A. A. Buchachenko, A. V. Stolyarov, M. M. Szczesniak, and G. Chalasinski, J. Chem. Phys. 137, 114305 (2012).

[33] B. P. Prascher, D. E. Woon, K. A. Peterson, T. H. Dunning Jr, and A. K. Wilson, Theor. Chem. Acc. 128, 69 (2011).

[34] I. Lim, P. Schwerdtfeger, B. Metz, and H. Stoll, J. Chem. Phys. 122, 104103 (2005).

[35] J. Č́́žek, J. Chem. Phys. 45, 4256 (1966).

[36] A. Derevianko, S. G. Porsev, and J. F. Babb, At. Data Nucl. Data Tables 96, 323 (2010).

[37] R. Moszynski, P. S. Żuchowski, and B. Jeziorski, Collect. Czech. Chem. Commun. 70, 1109 (2005).
[38] H.-J. Werner, P. J. Knowles, et al., "MOLPRO, version 2010.2: A package of ab initio programs," (2010), see http://www.molpro.net.

[39] T. Korona, M. Przybytek, and B. Jeziorski, Mol. Phys. 104, 2303 (2006).

[40] J. Deiglmayr, A. Grochola, M. Repp, O. Dulieu, R. Wester, and M. Weidemüller, Phys. Rev. A 82, 032503 (2010).

[41] M. Debatin, T. Takekoshi, R. Rameshan, L. Reichsollner, F. Ferlaino, R. Grimm, R. Vexiau, N. Bouloufa, O. Dulieu, and H.-C. Nagerl, Phys. Chem. Chem. Phys. 13, 18926 (2011).

[42] M. Urban and A. J. Sadlej, J. Chem. Phys. 103, 9692 (1995).

[43] M. Mayle, B. P. Ruzic, and J. L. Bohn, Phys. Rev. A 85, 062712 (2012).

[44] G. F. Gribakin and V. V. Flambaum, Phys. Rev. A 48, 546 (1993). 